Article title : Reconstructing the social constructionist view of emotions: from language to culture, including nonhuman culture

Author: Martin Aranguren

Journal for the Theory of Social Behavior 47 (2), pp. 244-260

Affiliation: Centre National de la Recherche Scientifique, Unité de Recherches Migrations et Sociétés, Paris 
Reconstructing the social constructionist view of emotions: from language to culture, including nonhuman culture

Abstract. The thesis of social constructionism is that emotions are shaped by culture and society. I build on this insight to show that existing social constructionist views of emotions, while providing valid research methods, overly restrict the scope of the social constructionist agenda. The restriction is due to the ontological assumption that social construction is indissociable from language. In the first part, I describe the details of the influential social constructionist views of Averill and Harré. Drawing on recent theorizing in psychology, I suggest that their fixation on language makes these approaches inadequate to the analysis of the social construction of human emotional experience. In the second part, I extend the argument to other species, suggesting that these social constructionist views are incapable of accommodating the fact, ascertained by primatologists, that animals have cultures, and that part of animal culture concerns the social molding of their emotions. I conclude that a reconstructed social constructionism should be regarded not as inimical to, but as part and parcel of, a nonreductive biology of emotions.

Keywords: affective sciences, animal culture, emotion, primatology, social constructionism 


\section{Reconstructing the social constructionist view of emotions From language to culture, including nonhuman culture}

The thesis of social constructionism is that emotions are shaped by culture and society. This sounds obvious to many sociologists and anthropologists familiar with the idea that phenomena such as gender or ethnicity are social constructions. To many psychologists and biologists with a bent for genetics and evolution theory, in contrast, this sounds suspicious.

I start with the intuition that both camps are right to some extent. I agree that emotions are molded by socio-cultural influences but I also think that there is something wrong with the usual way of dealing with social constructions. The overall aim of the article is to enlarge the scope of the social constructionist agenda in emotion research so as to make it relevant not only to unexplored areas of human emotions, but also to the emotions of nonhuman animals.

Social constructions can be regarded as those aspects in the lifestyle of a community which are not given but self-made and not genetically but socially transmitted. In this view, social constructions belong in the domain of culture, functionally defined as the social transmission of habits and knowledge. That emotions are socially constructed therefore means that emotions are cultural. Since this definition of culture does not presuppose any special ability to mentally represent the objects of cultural transmission, no mental representation of emotions is required for the latter to be socially constructed.

This is not to say that emotions are "just" cultural products, unless one endorses, unwittingly or not, the old dichotomy that sets culture in irreconciliable opposition to nature. It rather means that cultural influences concur, along with other factors such as physiological and cognitive processes, in the determination of concrete emotional phenomena.

In the context of this article emotions are conceptualized ecologically as modes of action readiness (Frijda, 1986). In common speech emotions are essentially inner "feelings," but the consensus today in the field of the affective sciences is that emotions are multi-componential phenomena. Alongside the experiential component (the "feeling"), this consensual view places a motor-expressive component and a 
physiological component. The epistemological implication is that emotions may be ascribed on the basis of self-reported experience, but also on the basis of observable expressive behaviors or measurable physiological changes. As will be suggested below, emotions are always expressed in behavior but the concomitant experience is not always verbally reportable. Therefore, the ability to report on inner feelings cannot be taken as the criterion for "validating" emotion ascriptions.

There are many definitions of culture, and the functional approach that I endorse contrasts with those that emphasize instead the medium or mechanism of transmission. In the latter view, unless the spreading proceeds through language, teaching, or imitation it cannot properly be said to be cultural. In contradistinction, from a functional point of view, culture is approached in terms of what it achieves. Beyond the broad specification that the method of transmission must be nongenetic to be cultural, the precise way in which the social spreading occurs is of secondary importance from this functional perspective.

In the first part of the article, I review the details of two influential social constructionist views of emotions, namely those of American psychologist Jim Averill and of British philosopher Rom Harré. Each of these views couples a set of ontological assumptions with a specific method for acquiring knowledge on the cultural dimension of emotions. The methods deserve all their place in the toolbox of emotion research, but the underlying ontology, by making social constructions indissociable from language, arguably impose unjustified limits to the agenda of social constructionism. As a result, the proposed methodologies, while fully adequate to their objects, address only part of a much broader domain. In this sense, I draw on recent theorizing in the psychology of emotions to argue that neither language nor specific emotion words are necessary for the social construction of emotional experience.

If the emotions of animals could be shown to be socially constructed, that would provide additional support to the claim that the social construction of emotions is not indissociable from the use of emotion words, but a process of much broader scope. This is what the second part of the article attempts to do. I first summarize evidence from ethnographic studies of animals suggesting that some nonhuman species have cultures. I anticipate that in the 1950 s primatology imported ethnography from the social sciences, a 
circumstance that makes this field intuitively appealing to sociologists and anthropologists familiar with the technique. After reviewing the ethnographic evidence for primate culture, I examine the possibility that the cultural accomplishments of animals may extend to the social molding of their emotions. Primatologists have found that macaques and chimpanzees reconciliate after fights, and have noted that this serves at once to alleviate fear and to repair social relations. I draw on this literature to suggest that the way in which primates cope with fear after fights is socially constructed.

\section{Social constructionist views of emotions}

This part reviews the influential social constructionist approaches of Averill and Harré. I take pains to render as faithfully as possible, within the space limits of this article, the nuances that distinguish one approach from the other. This interpretive exercise brings out a number of differences but also a crucial commonality. An important difference concerns the different methods that both authors propose. Harré's method, which he calls "Emotionology" (Harré, 2009), draws on the philosophy of ordinary language and is meant to deal with the local grammatical rules of use of emotion words. Averill's method borrows from sociology and is designed to treat emotions (or "emotional syndromes") as transitory social roles. This difference in method notwithstanding, I hope to show that both Harré and Averill make the domain of social construction indissociable from language, an ontological assumption that dramatically reduces the reach of the social constructionist agenda.

\section{Harré: Emotionology}

Harré (1987) saw his social constructionist approach to emotions as a part of a broader reformulation of the problématique of psychology. I endorse his suggestion that a paradigm is a combination of a metaphysical theory about the nature of the objects in a certain field (i.e. a "regional ontology") and a method for acquiring knowledge of those objects. 
What is Harré's regional ontology as far as emotions are concerned? His starting point is that "human conduct is the implementation of a belief system in the course of which language plays a distinctive and irreducible part." (Harré, 1987, p. 4). He asserts that behavior is invested with meaning by virtue of the intentions of actors and the interpretations of others, so that human action can be treated as a text. He goes on to propose that "social relations among human beings are created and maintained mainly by speech (and more recently by writing)" (Harré, 1987, p. 4). The epistemological implication is the familiar maxim of the sociology of Verstehen: the student of human action should search for semantic instead of causal relations; understanding action amounts to identifying the intention that the action expresses for the actor and for others.

The study of emotions is thus part of a broader program concerned with the semantic understanding of human action. In this context, emotions are not things out there that nouns could designate ("anger"), but actually qualifications of human action that are best captured by adverbs ("acting angrily"). In order to dispel this "ontological illusion" (Harré, 1987, p. 4), how are we to gain knowledge about emotions as qualifications of human action? In other words, what is Harré's method? The "Emotionology Principle" (Harré, 2009) is the answer. Following the lead of Austin and Wittgenstein, this principle asserts that understanding what an emotion is (say, anger) amounts to identifying the conditions for the proper use of the relevant word (that is, the grammar of the linguistic item “anger”).

The Emotionology Principle clarifies the sense in which Harré's approach is an instance of social constructionism. The "social" element is provided more precisely by the culturally shared and socially enforced rules for the correct use of words, which can be termed, for short, the grammar. The "construction" element visualizes emotions not as given sealed units but as the outcome of active processes of assembling lower-level components according to those rules. In the original statement of his view, Harré (1986) lists the following components as being involved in the orderly assemblage of emotions: a bodily agitation, an evaluative judgment, and the local moral order. In the 2009 restatement, however, the status of the local moral order changes. It is demoted from individual component to a modifier of other components, especially evaluative 
judgments. At the place that the moral order leaves empty, "a tendency to act" (Harré. 2009 , p. 295) is now plugged.

At any rate, the Emotionology Principle means that we can discover what an emotion is by unveiling the bodily agitation, the evaluative judgment, and the tendency to act that must be present in order for the relevant emotion word to be used correctly in the local culture. "Thus I would call myself 'angry' if I felt a certain kind of agitation, I thought that I had been unjustly injured in some way, and I had a tendency to respond to the situation in some rather strong manner." (Harré, 2009, p. 296) There is no anger; only the word "anger" and the specific agitation, the specific belief, and the specific response that the local grammar requires for the word "anger" to be properly used. Harré points out, however, that not all three classes of components are necessary for the correct use of all emotion words. He illustrates this remark with the examples of "loneliness" or "pride", which arguably do not involve any discernible physiological component (Harré, 1986).

If emotions are the physiological, cognitive, and behavioral conditions that must be met for emotion words to be properly applied, and local grammars stipulate which emotion words require which conditions, it follows that "there might be considerable cultural variety in the emotion repertoires of different peoples and epochs" (Harré, 1986, pp. 7-8). That is, the rich palette of emotion terms across cultures and historical periods cannot be reduced to a short list of allegedly primary, fundamental, or basic emotions. Each emotion word must be treated as the index of a unique set of conditions ${ }^{\mathrm{i}}$.

Averill: Role theory

Averill (1980, p. 313) declares that his approach "extends to the problem of emotion the type of inquiry traditionally known as the sociology of knowledge", after which he quotes Berger and Luckmann's 1966 classic The social construction of reality. In effect Averill's social constructionist view of emotion can be shown to rest on the following ontological claims by Berger and Luckmann: (1) nature is immutable (and therefore amenable to essentialistic thinking) while culture and society are self-produced and malleable; (2) humans are the only life form capable of entertaining a flexible or "open" relationship to their surroundings, so that self-produced culture and society are unique to 
humans; (3) language is the primary medium of the uniquely human process of production of culture and society.

Building on this ontology opposing nature's immutability to society's and culture's malleability, Averill's (1980, p. 305) opening proclamation is that "emotions are social constructions, not biological givens". For Averill, that emotions are social constructions means ipso facto that animals do not have emotions proper, since they lack the "open" relationship to the environment that makes social construction possible in the first place. Hence his belief "that the application of emotional concepts to animals is primarily metaphorical or derivative.” (Averill, 1980, p. 306) Finally, knowing how to apply emotional concepts as found in ordinary language is indispensable for experiencing emotions, insofar as it is "the categories of reflective thought that give an emotional experience its meaning" (Averill, 1980, p. 316).

What is Averill's method for acquiring knowledge on the social construction of emotions? The answer is role theory: emotions are to be seen as enactments of specific social roles with specific functions within the social system. Emotional social roles come in syndromes, and these syndromes are said to be constructed because they result from the assemblage of diverse somatic, mental, and socio-cultural components. The construction of these complex syndromes is said to be social because shared norms or expectancies "govern the selection of, and covariation among, various response elements" (Averill, 1980, p. 308). Social norms, by constituting social roles, constitute the complex sets of responses (that is, the syndromes) that we call emotions.

Averill points out that emotional syndromes are "polythetic", by which he means that they are not definable in terms of essential features. That is, there is no fixed list of specific somatic, mental, and socio-cultural phenomena that could provide the definition of any emotional concept, for example anger. Thus, "[t]here are certain behaviors that are typical of anger (including the way the individual appraises the situation), but none of these is necessary for the attribution of anger." (Averill, 1980, p. 307). In other words, there is no simple or complex event common to all instances of anger to which the term “anger" (or "happiness" or "envy") ultimately refers.

The enactment of a role, to be properly called emotional, must be experienced as a passion. More precisely, it must be interpreted, through the emotional categories provided 
by ordinary language, as a response beyond self-control. Averill postulates that an additional feature of emotional roles is the actor's appraisal of the situation, insofar as a definite evaluative attitude toward a given object is logically necessary to understand what we mean by this or that emotion concept. The same response, say some aggressive behavior, may be understood as anger or envy according to whether we see the actor as responding to an injustice, on the one hand, or to somebody else's access to some object that the actor values but cannot enjoy, on the other. We thus reach Averill's full definition of emotion from a role-theoretical perspective: "An emotion is a transitory social role (a socially constituted syndrome) that includes an individual's appraisal of the situation and that is interpreted as a passion rather than as an action." (Averill, 1980 , p. 312, author's italics). He emphasizes that these transitory social roles, and not inner feelings, are what emotion words symbolize.

Averill states that from this perspective there is an indefinite number of emotions, the only limit being the functional requirements of the social system. In his view, reducing the diversity of emotions recognized in ordinary language to a limited set of "basic" emotions is at best unilateral. In effect the concrete phenomena that emotion research encounters, Averill notes, are complex response syndromes that can be alternatively analyzed at the physiological, psychological, and socio-cultural levels. That is, there is no single level which is more "basic" than the others".

\section{The indissociability of social construction from language}

Apart from the difference in method between Emotionology and role theory, Harré and Averill diverge in the details of their understanding of the social construction of emotions. For Harré, social construction proceeds through the grammar of emotion words. The rules of use of specific emotion terms provide the principles whereby inherently disparate responses are assembled into unitary emotions. For Averill, in contrast, social construction stems from the functional requirements of the social system. Any social system relies on patterns of action for its continued existence, and social roles provide such patterns. The phenomena we call emotions are more fundamentally a special class of social roles that accomplish specific systemic functions. Emotion words 
are not visualized here as depending on a self-sufficient grammar, but as serving the symbolization of this special class of functional roles.

In both approaches, however, social construction is indissociable from language. In the case of Harré, this is sufficiently obvious. In the more tricky case of Averill, this is made apparent in his claim that the experience of emotion and the enactment of emotional social roles, as well as their recognition, require the use of linguistically expressed emotion concepts. That is, the social roles that emotions are supposed to be cannot exist without emotion concepts. In Averill's perspective, a socially constructed emotion for which no word is locally available makes no sense, since what is socially constructed are roles and these need to be linguistically represented for their performance and recognition.

This indissociability of language and social construction follows from the ontological framework of Harré's and Averill's respective social constructionisms. As we saw, Harré postulates that human conduct, including the special class of behaviors we call emotions, is the implementation of linguistically represented beliefs. In the case of Averill this can be made explicit again by consulting The social construction of reality. There Berger and Luckmann regard social constructions as "objectivations", that is, "products of human activity that are available both to their producers and to other men as elements of a common world" (Berger and Luckmann, 1966, p. 49) A couple of pages later, they note that " $[\mathrm{t}]$ he common objectivations of everyday life are maintained primarily by linguistic signification." (Berger and Luckmann, 1966, p. 51) I read this as stating that language is the primary medium whereby social constructions are kept in existence.

\section{Nonlinguistic but socially constructed emotional experience}

In the present state of knowledge in emotion research, perhaps the most obvious candidate area to social construction in which language plays a rather secondary role is emotional experience. Combining an information-processing model of psychological states with a phenomenological analysis of experience, Lambie and Marcel (2002) have proposed a theoretical framework that questions the centrality of language for the experience of emotion. The theory postulates first a distinction between emotion states 
and emotion experience. Following Frijda (1986), emotion states are conceptualized as modes of action readiness caused by appraisals of objects or situations. While all emotion states are assumed to entail some emotion experience (there is something all emotions are like), the particular content of the emotion experience cannot be deduced from the emotion state itself.

Drawing on a distinction classically elaborated by Sartre (1995), the authors conceptualize emotion experience as involving two possible types of consciousness, namely first- and second-order. Second-order, reflective emotion experience is consciousness of first-order, immediate emotion experience and is not a necessary accompaniment of emotion states. For any instance of emotion state, the particular content that defines the possible (but not necessary) second-order emotion experience depends on the way one attends to the necessary first-order emotion experience. Lambie and Marcel explain variability in attentional processes in terms of directionality (self/world) and mode of attention (synthetic/analytic, absorbed/detached). A third source of variability that is specific to emotion experience relates to whether attention is focused on appraisal or on action readiness. This framework accounts nicely for the varieties of experiential content characterizing emotion that have been documented so far in the literature, but most importantly for our purposes, it also defines reflective awareness of emotion, which is where emotion concepts fit, as only one among several alternative forms of emotion experience.

Challenging the assumption that the experience of emotion relies on the use of emotion concepts, two forms of unawareness of emotion can be conceptually distinguished on the basis of this framework. First, one may have no second-order awareness of one's emotion, the criterion of which is the inability to report on the emotion state combined with its observable nonverbal expression. Second, one may have second-order awareness of one's emotion but only insofar as individual components of experience are concerned, the criterion of which is the ability to report on the components ("I feel like running away", "My heart is beating hard") but the inability to subsume them under an emotion category such as "fear". In the first case of unawareness, the emotion state is expressed but unreportable. In the second case, the emotion state is reported not as a holistic emotion category, the only kind of report considered by Averill and Harré, 
but as the experience of isolated emotion components. This reporting of isolated emotion components could be referred to as "affect" talk, to differentiate it from verbalisations involving the use of emotion words proper such as "fear."

To sum up, all emotion states entail first-order emotion experience while only some of them also involve a second-order, linguistically represented experience of the relevant emotion. But even within the circumscribed class of emotional occurrences that happen to be experienced through the use of concepts, emotion categories such as "fear" are only part of a bigger picture. The reason is that second-order emotion experience need not involve holistic categories that bind together a variety of components ("fear"), but may limit itself to the analytical conceptualization of individual components ("I feel tense"), that is to "affect" talk. In this enlarged framework, not only are emotion words inessential to the experience of emotion: they are also inessential to the conceptualization of that experience.

Does this contingent relationship between the experience and the naming of emotions imply that emotion experience is not socially constructed? Not at all. It simply means that the locus of social shaping is no longer limited to the use of emotion words, but extends to the more fundamental attentional processes that make the use of emotion words possible in the first place. That is, we learn from others how to attend to our emotions, just as learning how to use our attentional abilities is part and parcel of the acquisition of any social practice. What in one culture may be experienced as a holistic emotion (say Schadenfreude), in the other may be analytically experienced as an addition of separate components (say "taking delight at the troubles of another", to use Harré's (2009, p. 299) own formula). And, of course, what in one culture may tend to enter linguistically represented, second-order experience (whether analytical or holistic), in the other may remain entirely unconceptualized as raw, first-order experience. The fact that culture influences attentional processes, and thereby the experience of emotions, does not exclude that there may also be interindividual, or sex, or age differences within the same culture, just as with any other social practice.

I conclude that there is no good reason to limit a priori the scope of the social construction of emotional experience to the grammar of emotion words. The qualification "a priori" stands as a reminder that the self-restriction applies as long as we hold the 
ontological assumption that the domain of social construction is indissociable from language. Once we drop this assumption, we open our theoretical eyes to forms of cultural molding of emotions in which language plays a secondary, and even nil role. We have just seen that language can be regarded as secondary in the social construction of emotional experience. In the next section of the article, I draw on the work of primatologists to suggest that in nonhuman animals the absence of language does not prevent them from socially constructing their emotions.

\section{The social construction of emotions in nonhuman primates}

In this section I first review some of the existing evidence that primatologists have gathered for the case of nonhuman cultures. Considering that primatology is little known among social scientists, the review offers some historical and epistemological background before addressing the issue of nonhuman culture. I then examine the possibility that nonhuman animals, as part of their cultural abilities, socially construct their emotions. I draw on research on conflict resolution among primates to suggest that reconciliation behavior is a socially constructed mode of emotional coping.

\section{Cultural primatology}

Japanese biologist and philosopher Kinji Imanishi is credited with having pioneered ethnographic methods in primatology in the early 1950s and having prepared the theoretical ground for the discovery of cultural phenomena among primates (de Waal, 2001). Owing to its own cultural background, Japanese primatology was arguably uncontaminated by the discontinuist assumptions of the Christian tradition and the related view that humans have souls but animals are mere automatons driven by instincts. In Eastern philosophies animals have souls too, so that that the body-soul dualism, instead of severing our species from other forms of life, actually connects us to them through the shared feature of spirituality ${ }^{\text {iii }}$.

The ethnographic study of primate groups promoted by Imanishi and his school relied first of all on the provisioning of food as a way of habituating the animals to the 
ethnographers' presence. Secondly, it involved long-term observation characterized by identification of individual members of the group and detailed description of their activities, with a special focus on social relations, including kinship relations, friendships, and rivalries.

The application of ethnography to the study of animal societies, which is now taken for granted, confronts the ethnographer with some of the problems that the epistemology of Verstehen is meant to address in the social sciences. Making sense of animal behavior, just as with humans, requires the ascription of beliefs and intentions to the behaving individuals. Consequently, in order to understand animal behavior, ethnographers make ample but controlled use of anthropomorphic terminology uniquely suited to the description of meaningful action. Thus the state in which an animal is may be described with the emotion word "fear" or the relationship between two animals with the term "dominance". This is to say that in the ethnography of animal behavior emotion terms have all their place.

The best-known evidence of animal culture brought to public attention by Imanishi's school of primatology came from the Japanese isle of Koshima in 1953. A juvenile female macaque invented the habit of carrying sweet potatoes to the water to clean off the dirt, and within a decade all the population below middle age had followed suit (de Waal, 1999). But not only did the pattern propagate: it also evolved. At the very beginning, monkeys used to wash potatoes in freshwater, but early on they changed to seawater, apparently to season the vegetable. Ever since, numerous such traditions have been documented across diverse behavioral areas (e.g. feeding, mating) not only in primates such as macaques and chimpanzees, but also in other mammals such as black rats and sea otters, and also in several bird species (McGrew, 1998). The list is not exhaustive.

In the late 1990s a group of primatologists from diverse origins published a comprehensive report on the similarities and differences in the behavioral repertoires of chimpanzees across seven field sites in Africa (Whiten et al., 1999). The two Eastern sites, located respectively in Guinea and Ivory Coast, dealt with a species called verus; the Western sites, two of which were in Uganda, and other two in Tanzania, with the species schweinfurtii. After establishing a list of behavior patterns suspected to vary across the sites (including different versions of the same pattern), the investigators 
assigned each behavior to one of six categories. The pattern was deemed to be "customary" if it occurred in all or most members of at least one age-sex class, "habitual" if it was not customary but occurred repeatedly in several individuals, "present" if it was neither customary nor habitual but clearly identified, and "absent" if it had not been recorded and no ecological explanation was available. "Ecological explanation", in turn, was used for those behaviors whose absence was explicable because of a local ecological feature. For example, and ecological explanation of the absence of algae-fishing within a community is to point to the rarity of algae as its cause. The residual category was labeled "unknown".

39 patterns of behavior were found to vary across chimpanzee communities, that is, they were customary or habitual in some sites but absent in others. Some of them were unique to certain communities, but most were shared between two or more communities. In addition, the patterns varied as much between groups of the same species as between verus (in the West) and schweifurthii (in the East). However, nut-cracking, another famous chimpanzee feat, was observed only in the Eastern sites. For this complex behavior pattern chimpanzees use a hammer and an anvil made of either wood or stone to pound dried palm nuts so as to break them open.

The primatologists argue that neither genes nor ecological factors, but social learning alone provides the key to the spreading of potato washing among the macaques of Koshima and to the variability in chimpanzee behavior across the African sites. From the premise that culture is transmission through social means, they conclude that these are instances of nonhuman cultures.

However, this argument encounters two lines of opposition that must be briefly stated because the debate is far from being clinched. On the one hand, we find those who would counter, in typical social constructionist manner, that culture requires language. A second, more challenging line of opposition comes from experimental psychologists who argue that culture involves specific learning mechanisms, namely imitation and teaching, that rest on higher cognitive abilities which have been found so far only in humans (Galef, 1992; Tomasello, Kruger, and Ratner, 1993). The primatologists have replied from a functional standpoint that it is not the mechanism but the achievement of spreading through social learning that defines culture (e.g. de Waal, 1999). 


\section{The social construction of macaque emotions}

The literature on reconciliation in a variety of primate species provides some empirical basis to the suggestion that the emotions of animals too are to some extent culturally constructed (for a comprehensive review, cf. de Waal, 2000). Primatologists define reconciliation as a friendly reunion between former opponents of the same species not long after an aggressive confrontation. According to species, the friendly reunion is accomplished through a variety of behaviors such as smacking the lips, presenting the genitals, or displaying a play-face, and it appears to serve a calming function and to repair damaged social relationships. They usually occur within three minutes after conflict termination.

Aureli, van Schaik, and van Hoof (1989) conducted a study on long-tailed macaques (Macaca fascicularis) to test the hypothesis that reconciliation should lead to a faster reduction of anxiety than if no reconciliation had taken place after a conflict. They also hypothesized that the loser, fearing a repeated attack by the winner, should be the one to seek the reconciliation. Building on other studies suggesting that scratching is a good indicator of sympathetic arousal they used the rate of scratching of the animal to measure anxiety. Scratching was recorded for each individual involved in a conflict (i) during the ten minutes following the confrontation and (ii) again the next day at the same time in a non conflict situation ("matched-control").

The authors compared the rate of scratching observed in three separate conditions, namely post-conflict without reconciliation, post-conflict with reconciliation, and "matched-control". Their unequivocal conclusion was that "reconciliation actually caused the fast stress reduction" (Aureli, van Schaik, and van Hooff, 1989, p. 44). In the postconflict without reconciliation condition, the rate of scratching returned to normal within about five minutes, with the first three minutes featuring the highest rates of scratching. These levels were nearly the same in the post-conflict with reconciliation condition, but only during the short period between the end of the conflict and the beginning of the friendly reunion. In this condition, once the reconciliation took place the rate of scratching not only declined but also fell below the confidence interval of the control 
measure. The authors also found that the relative frequency leading to friendly reunions was skewed toward the loser versus the winner, but only during the first three minutes following the confrontation. In this special situation, losers took the initiative in $75 \%$ of the cases, but in all other contexts the majority of initiatives came from the winner.

The emerging picture is one in which, following conflict, macaque losers initiate reconciliation in order to appease the fear of being attacked again by the winner. To put it in the terms of the human psychology of emotions, it can be said that macaques "cope with fear" through reconciliation. The question that I wish to raise now is whether coping, a mode of behavior that psychologists acknowledge to be integral to emotion (Lazarus, 1991), is genetically inherited or socially transmitted in macaques. There is some indication that the latter is the case, suggesting that animal emotional coping, as the human variety, is not impermeable to cultural influences.

de Waal and Johanowicz (1993) conducted an experiment to assess whether reconciliation in macaques is a socially learnt behavior pattern. In order to test this hypothesis, they begun with a remarkable contrast previously found in the reconciliation behavior of two closely related macaque species, namely stumptail monkeys (Macaca arctoides) and rhesus monkeys (Macaca mulatta). Having a generally more tolerant and less antagonistic style of social life, stumptail monkeys reconcile in a much larger proportion than rhesus monkeys. Building on this difference, the authors devised a procedure to test whether exposure to older stumptail monkeys increased the reconciliation rate of young rhesus monkeys, as compared to a control group of rhesus monkeys exposed only to older conspecifics. The rationale was that the rhesus subjects should be receptive to the environmental influence of the stumptail models, since rhesus appear to differ from stumptail monkeys only in degree and not in terms of basic behavioral tendencies and social cognition.

Reconciliation rate was defined as the percentage of agonistic conflicts in which the subject was involved followed within 3 minutes by a reconciliation initiated by the subject. In contrast with the Aureli et al study reported above, in this experiment no difference was made between the winner or the loser of the conflict. The procedure involved three successive phases. In the Prephase rhesus subjects and controls lived only with conspecifics for 3 weeks. In the Cohousing phase, spanning across 22.5 weeks, 
rhesus subjects were placed together with stumptail macaques, while rhesus controls remained with conspecifics. In the Postphase the species were finally segregated for 6 weeks.

The results showed that the tendency to reconciliate of individual rhesus subjects (i.e. those that were placed together with stumptail monkeys during the Cohousing phase) increased from $10 \%$ in the Prephase to $30 \%$ in the Postphase, while the same tendency for rhesus controls remained stable around 10\% throughout. In the Postphase, this 30\% reconciliation rate remained stable despite the fact that subject rhesus monkeys were housed again with less conciliatory conspecifics. According to the authors, the gap between the subjects and the controls reflects a more general interspecific difference, namely that stumptail monkeys reconciliate three to four times more often than rhesus monkeys. de Waal and Johanowicz thus concluded that reconciliation behavior is modifiable through social learning.

Aureli et al. (1989) found support for the hypothesis that reconciliation among longtailed macaques is a mode of coping with fear. de Waal and Johanowicz (1993) produced evidence indicating that reconciliation among rhesus macaques is subject to social influences. This provides some basis for the hypothesis that coping with fear, as an integral function of reconciliation, is subject to social influences among macaques. That is, coping with fear is socially molded, which is another way of saying that it is socially constructed.

Three qualifications are in order. First, it is important to keep in mind that the study on the coping function of reconciliation concerned stumptail macaques, while the evidence on the social modifiability of reconciliation behavior came from rhesus macaques. In this regard, I recall that it is customary in ethology to draw parallels between different species of the same genus. Indeed the reported experiment by de Waal and Johanowicz (1993) rests on the assumption that such parallels are both theoretically valid and heuristically fruitful. Second, the reader may be reminded that the studies reported in this section did not use the concepts of "coping with fear" and "social construction" but rather of "stress reduction" and "social modifiability." This being said, I do not think that the change in terminology distorts the interpretation provided by the authors, although the modified vocabulary does of course change its connotations. Third, even though the authors of the 
reported primate studies did not worry very much about ascribing emotions to nonhuman animals, some readers of the present article might. This raises two interrelated questions.

On the one hand, what is the criterion for ascribing emotions to nonhuman primates? Instead of considering emotions in general and in the abstract, the question is best addressed by focusing on the ascription criteria of specific emotions, and by looking at what primatologists actually do in their scientific practice. Bethell, Holmes, MacLarnon, and Semple (2012), for example, conducted an experiment to investigate whether emotion, and in particular anxiety, mediates social attention among rhesus macaques. Their method for ascribing anxiety to macaques involved two components: first, a situation assumed to be stressful for macaques, namely a veterinary health check involving physical restraint and injection; second, movements assumed to express anxiety, such as self-directed, stereotypical and self-injurious behaviors. As with humans, the behaviors that strike as prima facie expressions of emotions are characterized by their departure from the requirements of instrumental interaction with the environment (Frijda, 1986). Thus, in the context of the stressful veterinary check, researchers interpreted some noninstrumental behaviors exhibited by macaques as expressions of anxiety.

The hypothesis of emotion has epistemic added value because it moderates the relationship between the external situation and the external response of the macaques. Emotion is the internal state that results from the way in which macaques appraise the veterinary check and that motivates them to perform self-directed, stereotypical and selfinjurious behaviors. It is by virtue of the hypothesis of emotion that the veterinary check can be said to be stressful and that the noninstrumental behaviors can be said to be expressive of anxiety.

But on the other hand, and more fundamentally, does it make sense to ascribe emotions to primates at all? I am inclined to reply in the affirmative as long as the hypothesis of emotion provides a theoretically parsimonious and empirically adequate way of making sense of nonhuman primates' behavior. If this attitude can be accused of anthropomorphism, the opposite view can be charged of anthropocentrism. Why assume that we are so different from our phylogenetic cousins that only our expressive behavior, but not theirs, could be interpreted as motivated by emotion in response to a situational configuration? Actually, from an evolutionary point of view the burden of proof is with 
the anthropocentrists, not with us anthropomorphists. In order to set the ontological stage for the epistemological objection that ascribing emotions to animals is metaphor at best, and nonsense at worse, they should convince us that despite the fact of common ancestry, we have emotions but animals do not.

Last, and still more fundamentally, does it make sense to account for the behavior of an animal, human or other, in terms of emotions at all? Various forms of behaviorism, in frontal opposition to the cognitivist orientation nowadays dominant in psychology, have been historically hostile to granting explanatory status to mental states such as beliefs or emotions in the analysis of behavior. So the general answer hinges on the reader's sympathy for the methodological and ontological tenets of behaviorism. But more specifically with regard to the argument to the effect that macaque emotions are socially constructed, I find it hard to understand the behavior of macaque losers after conflict without positing an internal state such as anxiety or fear. Why would a macaque loser dramatically increase the rate of scratching in such a context? The desideratum of behaviorism is to explain behavior in terms of external sources solely, and indeed many behavioral phenomena can be accounted for satisfactorily with reference to situational requirements. But in what sense would the situation of defeat demand that the loser scratches more intensely? Presumably, the primatologists reasoned that since there is no intelligible link between what the situation demands and what the macaque loser is doing, the behavior should be understood not as a response to the requirements of the external situation but as an effect of an internal cause named anxiety.

Again, it is the breakdown of the instrumentalist interpretation of behavior that motivates the hypothesis of emotion in the first place. In this context, pressing the analyst to discard all imaginable purely "behavioral" explanations before proposing an account in terms of internal states such as emotions presupposes the behaviorist view according to which external explanations are good and internal explanations are bad. Readers who do not feel committed to behaviorism, as much as the primatologists whose work I have reported, will not find anything special in the recourse to emotion in the explanation of behavior not obviously instrumental. Outside of behaviorist circles, from ethology to anthropology, this is just common scientific practice. 


\section{Conclusion}

In this article I have attempted to make a constructive revision of some aspects of the social constructionist view of emotions. I distinguished the methodology from the ontology of social constructionism to suggest that the methods should be held while the ontological background should be reconsidered. This ontological basis binds social construction and language together in such a way that important areas of social construction cannot be adequately dealt with. One such area is emotional experience, which can be regarded as socially molded but only secondarily concerned with language and even more so with emotion words. Another critical area of nonlinguistic social construction are the emotions of nonhuman animals, and more specifically the techniques for coping with fear that individual animals learn from others.

If even the emotions of nonhuman primates are susceptible of cultural analysis, maybe it is time to revise the dichotomy of nature and culture on which classical social constructionism is predicated. The paradoxical conclusion is that in this broader framework the social constructionist problématique is not the opposite, but actually part and parcel of a nonreductive biology of emotions. 


\section{References}

Aureli, F., van Schaik C. P., \& van Hooff J.A. (1989). Functional aspects of reconciliation among captive long-tailed macaques (Macaca Fascicularis). American Journal of Primatology, 19, 39-51.

Averill, J. R. (1980). A constructivist view of emotion. In R. Plutchik and H. Kellerman (Eds.), Emotion: Theory Research, and Experience, 305-39. San Diego (California): Academic Press.

Berger, P. L., \& Luckmann T. (1966). The social construction of reality: a treatise in the sociology of knowledge, Garden City, NY: Anchor Books.

Bethell, E., Holmes A., MacLarnon A. \& Semple S. (2012). Evidence that emotion mediates social attention in rhesus macaques. Plos One, 7(12).

Descola, P. (2005). Par-delà nature et culture. Paris: Gallimard.

de Waal, F. (1999). Cultural primatology comes of age, Nature 399, 635-36.

de Waal, F. (2000). Primates: a natural history of conflict resolution. Science 289: 58690.

de Waal, F. (2001). The ape and the sushi master: cultural reflections of a primatologist, New York: Basic books.

de Waal, F. B., \& Johanowicz, D. L. (1993). Modification of reconciliation behavior through social experience: an experiment with two macaque species. Child development, 64, 897-908.

Frijda, N. (1986). The emotions. London: Cambridge University Press.

Galef, B. G. (1992). The question of animal culture. Human nature 3 (2): 157-78.

Harré, R. (1986). An outline of the social constructionist viewpoint. In R. Harré (Ed.), The social construction of emotions (pp. 2-14), Oxford (England): Basil Blackwell.

Harré, R. (1987). Enlarging the paradigm. New ideas in psychology, 5 (1), 3-12.

Harré, R. (2009). Emotions as Cognitive-Affective-Somatic Hybrids. Emotion Review 1 (4), 294-301.

Lambie, J. A., \& Marcel, A. J. (2002). Consciousness and the varieties of emotion experience: a theoretical framework. Psychological review, 109 (2), 219-59.

Lazarus, R. S. (1991). Emotion and adaptation. New York: Oxford University Press.

McGrew, W. C. (1998). Culture in nonhuman primates? Annual review of anthropology, 27, 301-28.

Sartre, J.-P. (1995). Esquisse d'une théorie des émotions. Paris: Hermann.

Tomasello, M., Kruger, A. C., \& Ratner, H. H. (1993). "Cultural Learning.” Behavioral and brain science, 16, 495-552.

Whiten, A., Goodall, J., McGrew, W. C., Nishida, T., Reynolds, V., Sugiyama, Y., Tutin C. E., Wrangham, R. W., \& Boesch C. (1999). Cultures in chimpanzees. Nature, $399,682-85$.

\section{Acknowledgements}


I thank Brian Parkinson and Dominique Vidal for their generous and critical reading of an early version of this article, and three anonymous reviewers for two rounds of sharp and stimulating comments on the manuscript that I submitted to the journal.

${ }^{\mathrm{i}}$ In the 2009 restatement, however, exceptions are admitted to the claim that each emotion term has a unique and irreducible grammar. Harré introduces in effect the wittgensteinian notion of "hinges" to explore the higher order rules of use that are common to distinct emotion words such as "anger" and "jealousy". In the search for "hinges", emotion words are lumped together instead of being treated as unique.

ii It is important to note that Averill strategically mobilizes this stratified conception of behavior, which he derives from the work of Talcott Parsons, to deal with the particular problem of basic emotions. It is unclear whether or to what extent such framework is compatible with the ontological background that his social constructionist view of emotion inherits from Berger and Luckmann.

iii This is, of course, the "ontology" that French anthropologist Philippe Descola (2005) designates with the term animism. 